\title{
(65) Cybele: detection of small silicate grains, water-ice, and organics
}

\author{
J. Licandro ${ }^{1,2}$, H. Campins ${ }^{3}$, M. Kelley ${ }^{4}$, K. Hargrove ${ }^{3}$, N. Pinilla-Alonso ${ }^{5}$, D. Cruikshank ${ }^{5}$, \\ A. S. Rivkin ${ }^{6}$, and J. Emery ${ }^{7}$
}

\author{
${ }^{1}$ Instituto de Astrofísica de Canarias, c/vía Láctea s/n, 38200 La Laguna, Tenerife, Spain \\ e-mail: jlicandr@iac.es \\ 2 Departamento de Astrofísica, Universidad de La Laguna, 38205 La Laguna, Tenerife, Spain \\ 3 Physics Department, University of Central Florida, Orlando, FL 32816, USA \\ ${ }^{4}$ Department of Astronomy, University of Maryland, College Park, MD 20742-2421, USA \\ 5 NASA Ames Research Center, Moffett Field, CA, USA \\ 6 Johns Hopkins University Applied Physics Laboratory, Laurel, Maryland 20723, USA \\ 7 Earth and Planetary Science Department, University of Tennessee, Knoxville, Tennessee 37996, USA
}

Received 5 July 2010 / Accepted 9 September 2010

\begin{abstract}
Context. (65) Cybele is the most representative member of a population of primitive asteroids in the outer edge of the main belt, the Cybele asteroids. Recent dynamical models suggest that a significant fraction of them originated in the primordial transneptunian disk, so the study of the physical properties of these asteroids is potentially a useful test of these models.

Aims. Our aim is to obtain information on the surface composition of this asteroid. In particular we want to obtain information on the composition and properties of the regolith and the possible presence of ices and organic materials.

Methods. We present 2-4 $\mu \mathrm{m}$ and 5-14 $\mu \mathrm{m}$ spectroscopy of (65) Cybele obtained with the NASA IRTF telescope and Spitzer Space Telescope respectively. We compare the results with spectra of Trojan asteroids and asteroid (24) Themis. We analyze the $2-4 \mu \mathrm{m}$ spectrum using scattering models and we apply thermal models to the 5-14 $\mu \mathrm{m}$ data.

Results. The 2-4 $\mu \mathrm{m}$ spectrum of (65) Cybele presents an absorption band centered at $\sim 3.1 \mu \mathrm{m}$ and more weaker bands in the 3.2-3.6 $\mu \mathrm{m}$ region, very similar to those observed in (24) Themis. No hydrated silicates are detected. From the spectrum in the 5-14 $\mu \mathrm{m}$ region an effective diameter $D=290 \pm 5 \mathrm{~km}$, a beaming paramete $\eta=0.967 \pm 0.014$, and a geometric visible albedo $p_{V}=0.05 \pm 0.01$ are derived using the NEATM thermal model. The emisivity spectrum in the 5-14 $\mu \mathrm{m}$ range exhibits an emission plateau at about 9 to $12 \mu \mathrm{m}$ with an spectral contrast of $\sim 5 \%$. This emission is similar to that of Trojan asteroids and active comets and may be due to small silicate grains being imbedded in a relatively transparent matrix, or to a very under-dense (fairy-castle) surface structure. The lower amplitude of the silicate emission in Cybele's spectrum with respect to that of Trojan asteroids could be attributed to larger dust particles and/or a slightly denser structure.

Conclusions. The surface of (65) Cybele is covered by a fine anhydrous silicate grains mantle, with a small amount of water ice and complex organic solids. This is similar to comet surface where non-equilibrium phases coexist. The presence of water-ice and anhydrous silicates is indicative that hydration did not happened or is incomplete, suggesting that the temperatures were always sufficiently low.
\end{abstract}

Key words. minor planets, asteroids: general - minor planets, asteroids: individual: (65) Cybele - comets: general methods: observational - techniques: spectroscopic

\section{Introduction}

Outer main-belt asteroid (65) Cybele (hereafter Cybele) is one of the 10 largest asteroids (effective diameter $D=273 \pm 11.9 \mathrm{~km}$, Müller \& Bloemmaert 2004) and is representative of one of the populations in the outer edge of the main belt, the Cybele asteroids. Photometry and spectroscopy in the visible show that it is primitive. It has received different spectral classifications from various authors: P-type (Tholen 1989), C0-type (Barucci et al. 1987), Xc-type (Bus 1999), C-type (Lazzaro et al. 2004), and Xk-type (DeMeo et al. 2009). The spectrum, featureless longward of $0.5 \mu \mathrm{m}$ with a spectral slope $S_{V}^{\prime}=$ $0.95 \% / 1000 \AA$ (Licandro et al. 2008) and its low albedo $\left(p_{V} \sim\right.$ 0.05 , Müller \& Bloemmaert 2004) confirm that it is a primitive asteroid that belong to the C-complex defined by Tholen (1989).
The asteroids in the outer belt, which are here defined as objects with a semi-major axis $a>3.3 \mathrm{AU}$, fall into three main groups: the Cybeles with $a$ between 3.3 to 3.7 AU, the Hildas in the 3:2 resonance with Jupiter at $\sim 4.0 \mathrm{AU}$, and the Jupiter Trojans around the L4 and L5 Lagrangian points of Jupiter at 75.2 AU. Due to their large heliocentric distances, the outer belt asteroids have experienced less heating and are expected to be of a more primitive composition. They may also contain a significant fraction of ice in their interiors (Jones et al. 1990). Thus, these objects may provide a number of clues to the origin and evolution of the asteroid belt and to the formation of our planetary system. The origin of these populations is under debate. Until 2005, Trojans were considered as planetesimals formed in the vicinity of Jupiter's orbit and captured in the L4 and L5 Lagrangian points, but recent numerical modeling suggests 
they originated in the primordial transneptunian disk (Morbidelli et al. 2005). Recently, Levison et al. (2009) suggested that a significant fraction of the members of all outer main belt populations are TNOs moved to these resonances in an early epoch of the Solar System called the late heavy bombardment.

The study of the physical properties of these asteroid populations provide tests of dynamical models. In particular it is very important to compare them with that of Jupiter family comets, as the latter are small TNOs scattered to the inner planetary region (Fernández 1980). At visible wavelengths, Trojan, Hilda and Cybele asteroids are classified primarily as D- and P-type asteroids, with a small fraction of C-type. The color distribution of these populations indicate that the fraction of C-type asteroids decreases with increasing $a$. The few properly observed cometary nuclei also have D-, or P-type spectra (Licandro et al. 2002; Abell et al. 2005; Campins et al. 2006, 2007). C-type asteroids are the dominant spectral type of asteroids in the $3 \mathrm{AU}$ region. They are spectrally neutral in the visible and near-infrared wavelengths (0.35 to $2.4 \mu \mathrm{m})$ with a downturn in reflectance shortward of $0.4 \mu \mathrm{m}$ (Tholen 1989) and have low albedos. Dand P-type asteroids are characterized by low albedos and featureless reddish spectral curves in the visible and near-infrared. P-type objects are less red than D-types.

Some of the materials that have been suggested to be present in the surface of primitive asteroids have deep absorption bands in the $\mathrm{L}$ band around $3 \mu \mathrm{m}$ : hydrated silicates, complex organics, and ices. While the spectra of C-, P-, D-type asteroids in the $0.5-2.5 \mu \mathrm{m}$ region do not present any deep absorption band, even a small fraction of hydrated silicates, organics and/or ices in their surface should produce a detectable absorption band in a high $S / N 2-4 \mu \mathrm{m}$ spectrum (see Emery \& Brown 2004). Only a few primitive asteroids have been observed in the $2-4 \mu \mathrm{m}$ region, and most of them at a low $S / N \sim 10$ level. Absorption features are detected in the spectra of many C-types (Lebofsky 1980; Jones et al. 1990; Rivkin et al. 2002), consistent with the presence of a significant fraction of hydrated minerals. Water ice and organics were recently detected using 2-4 $\mu \mathrm{m}$ spectroscopy in the surface of C-type asteroid (24) Themis (hereafter Themis, Campins et al. 2010; Rivkin \& Emery 2010), an asteroid in the edge of the main belt, with $a=3.13 \mathrm{AU}$. Themis is the largest member of a collisional family that has 2 members that presented comet-like activity according to Hsieh \& Jewitt (2006). Most of the P-, and D-type asteroids observed in the $3 \mu \mathrm{m}$ spectral region are Trojans. Emery \& Brown (2003) observed 8 Trojan asteroids in the $K$ and $L$ bands, they did not detect any absorption band, and conclude that the spectra are compatible with silicate surfaces mixed with a significant fraction of low albedo material. But the $S / N$ of the Emery \& Brown spectra is too low to detect the $3.1 \mu \mathrm{m}$ absorption band reported in the spectrum of Themis and attributed to water ice and organics. So, the presence in outer belt asteroids of absorption features due to hydrated minerals, ice, or organics, similar in strength to that observed in 24 Themis, is not constrained by the available observations.

The mid-infrared spectral region is also proving to be diagnostic of the surface composition of primitive asteroids, and in ways complementary to the shorter wavelengths (e.g., Cohen et al. 1998; Müller \& Blommaert 2004; Lim et al. 2005; Emery et al. 2006; Campins et al. 2007). The three published midinfrared spectra, from 5 to $38 \mu \mathrm{m}$ of Trojan asteroids (Emery et al. 2006) exhibit clear emissivity features from which the compositional and physical properties can be inferred. Spectra of these three Trojans strongly resemble one another and are similar to the spectra of active comets. They present an emission plateau at about 9.1 to $11.5 \mu \mathrm{m}$ with a spectral contrast of
10 to $15 \%$. At these wavelengths, silicates show an emissivity high near $10 \mu \mathrm{m}$ (the Si-O stretch fundamental). The major features of the Trojan asteroid spectra can be reproduced using only fine-grained silicates (Emery et al. 2006).

Cybele asteroids are particularly important because they are a transition group between the primitive inner main belt asteroids and the Trojans, both of which are better studied populations with significantly different characteristics.

In this paper we present near-infrared 2-4 $\mu \mathrm{m}$ and midinfrared 5-14 $\mu \mathrm{m}$ spectroscopy of Cybele obtained with the NASA IRTF telescope and the Spitzer space observatory, and characterize its surface composition and other properties such as radius and albedo. In Sect. 2 the Spitzer and IRTF observations and data reduction are presented. In Sect. 3 the thermal model applied to the Spitzer spectrum and the derived size and albedo are presented. In Sect. 4 the emissivity spectrum is presented and costraints on the silicate surface composition are derived. In Sect. 5 the $2-4 \mu \mathrm{m}$ spectrum is presented. In Sect. 6 a discussion of the results is presented and the conclusions are summarized.

\section{Observations and data reduction}

Cybele was observed with Spitzer using the IRS spectrograph on February 8, 2005 and with the NASA 3.5 m IRTF telescope using the SPEX spectrograph on September 8 and 9, 2009.

\subsection{Spitzer mid infrared spectrum}

The spectrum of Cybele was obtained with the Infrared Spectrograph (IRS) instrument (Houck et al. 2004) on NASA Spitzer Space Telescope (Werner et al. 2004), on Feb. 8, 2005 UT. The asteroid was observed at a heliocentric distance of 3.296 AU, at a Spitzer distance of 3.474 AU, a phase angle of $-14.2^{\circ}$ (the phase angle is negative to indicate that the observation occurred before opposition). The IRS measures spectra over the range 5.2-38 $\mu \mathrm{m}$ with several different configurations. The observations presented here used two segments of the low spectral resolution mode $(R=\lambda / \Delta \lambda \sim 64-128)$. These two segments are short wavelength, 2nd order (SL2; 5.2 to $7.6 \mu \mathrm{m}$ ), short wavelength, 1 st order (SL1; 7.4 to $14.2 \mu \mathrm{m}$ ). When one of the low-resolution orders is centered on the target, the other is offset $\left(\sim 50^{\prime \prime}\right)$ from the target, providing a measurement of the sky background. Our analysis is limited to wavelengths $<13.2 \mu \mathrm{m}$ to avoid a known artifact in IRS spectra at 13.2-14 $\mu \mathrm{m}$ (the $14 \mu \mathrm{m}$ teardrop). The final spectrum corresponds to a total on-object exposure time of $29.4 \mathrm{~s}$ and $12.6 \mathrm{~s}$ for the SL2 and SL1 modes, respectively.

Our reduction begins with the basic calibrated data from the Spitzer Science Center's IRS reduction pipeline version S18.7.0. We background subtract and bad pixel mask the images, then extract spectra using the SPICE (Spitzer IRS Custom Extraction) software, using the default point source-tuned aperture. We scaled the SL2 spectrum by 0.92 to match the SL1 spectrum at $7.5 \mu \mathrm{m}$. This scale factor, although quite different from 1.0 , is consistent with the IRS pipeline's absolute calibration uncertainty of $\leq 10 \%$. When reporting values that depend on the absolute calibration (e.g., thermal emission model parameters), we will include an additional 10\% flux uncertainty added in quadrature. The flux density spectrum is plotted in Fig. 1 along with the best-fit thermal model (see Sect. 3). 


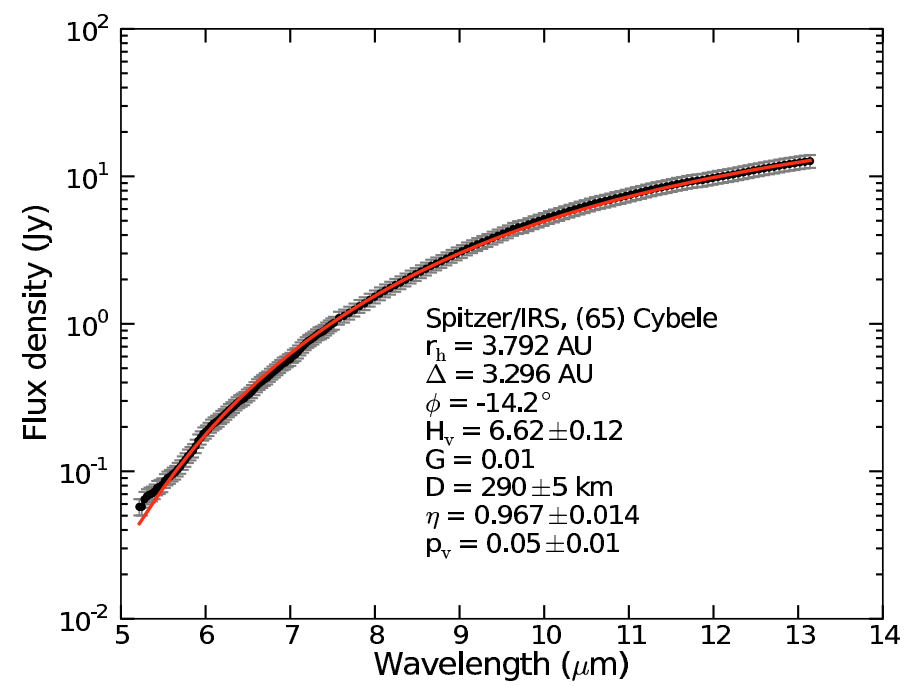

Fig. 1. Flux density spectrum of Cybele in the mid-infrared (points) and best-fit NEATM (red line).

\subsection{IRTF LXD spectra}

Near-infrared spectra of Cybele were obtained using NASAs IRTF on September 8 and 9, 2009 UT. The heliocentric and geocentric distances were 3.37 and $2.36 \mathrm{AU}$, respectively, and the phase angle was 0.6 degrees. We used the SpeX spectrograph/imager (Rayner et al. 1998) in the Long Cross-dispersed (LXD) mode to record spectra that each had a wavelength range from 1.95 to $4.0 \mu \mathrm{m}$. The slit was the $0.8 \times 15$ arcsec one, with an image scale of $0.15 \mathrm{arcsec} / \mathrm{pixel}$; this provided a spectral resolution $\lambda / \Delta \lambda \sim 800$, which we later binned to a dispersion of $0.01 \mu \mathrm{m}(\lambda / \Delta \lambda \sim 300)$ to maximize the signal-to-noise while preserving an appropriate spectral resolution. Spectra were obtained in pairs, labeled $\mathrm{A}$ and $\mathrm{B}$, offsetting the telescope by 10 arcsec along the slit between the two. The reduction and calibration of our spectra was done with the Spextool program (Cushing et al. 2004). Detailed descriptions of the reduction process can be found in Volquardsen et al. (2007). Briefly, the difference of each $\mathrm{AB}$ pair was taken to remove the sky emission, the resulting frames were then registered, flat-fielded, and 1-D spectra were extracted. Wavelength calibration was performed by matching arc lines to a model of arc lines vs. wavelength in Spextool. Solar analogue stars 51 Pegasus and HD 42618, and G Landolt stars (Landolt 1992) SA113_274 and SA115_271 were observed along the night covering similar airmasses as the asteroid and used to correct for telluric absorptions and to obtain the reflectance spectrum of Cybele. Telluric features were removed from extracted spectra of the asteroid and the solar analogs using a model atmospheric transmission profile called ATRAN (Lord 1992), which was generated for the zenith distance of the object, altitude of the telescope, spectral resolution, and a range of precipitable water vapor amounts. Outside the strongest telluric band (2.45 to $2.85 \mu \mathrm{m}$, which we omit) the random errors range from $3 \%$ to $10 \%$ for each point, with the lowest uncertainties at the shortest wavelengths; the point-to-point variability within each spectrum confirms this. We obtained a total of 120 and $88 \mathrm{AB}$ pairs of spectra over the first and second night respectively, and binned them into several separate time intervals to facilitate searching for rotational variation (see Fig. 2). In both nights the asteroid was observed in an interval larger that its rotation period (4.04 h, Gil-Hutton 1990). Within an interval, all spectra were averaged together.
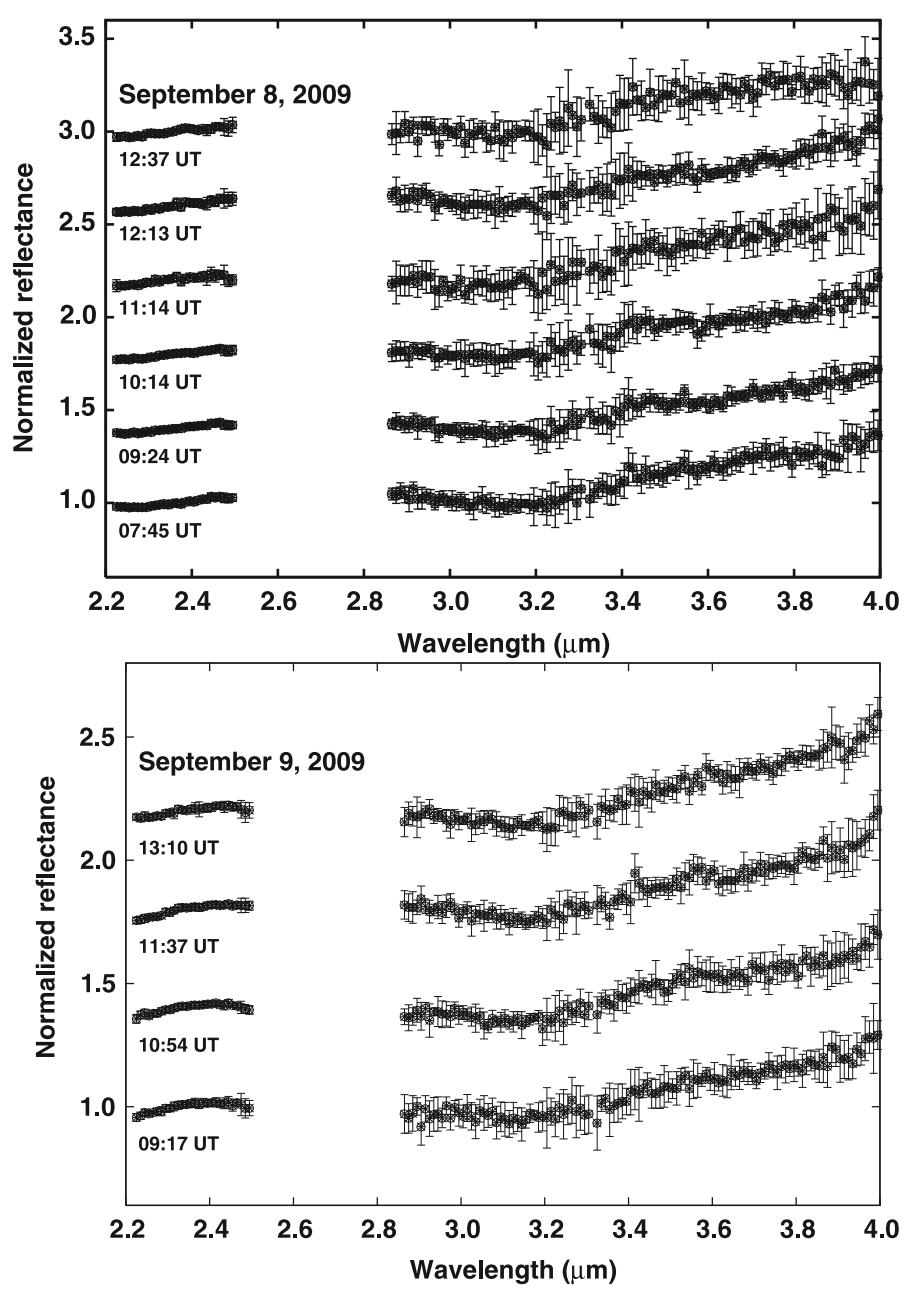

Fig. 2. IRTF reflectance spectra of Cybele normalized at $2.4 \mu \mathrm{m}$, obtained in both nights and shifted in reflectance for clarity.

To check for possible rotational variation of the asteroid spectrum due to surface inhomogeneities, each spectrum is divided by the average of all the spectra obtained each night (see Fig. 3). There is no statistically significant variation of the spectra, in particular of the observed absorption band at $3.1 \mu \mathrm{m}$, which preserves its shape and depth throughout. Comparing also the mean spectrum of Sept. 8 and 9 (see Fig. 4) we also notice that there are no significant variation between them, only a small difference in the spectral slope much smaller than the typical slope uncertainty $(\sim 1-2 \% / 0.1 \mu \mathrm{m})$.

\section{Thermal model}

The flux from Cybele in the wavelength range covered by the IRS is dominated by thermal emission. The measured spectral energy distribution (SED) depends on the object's size, composition, and temperature distribution. This last term is dependent on several factors, including distance from the Sun, albedo, thermal inertia, surface roughness, rotation rate, shape, and spinpole orientation. We used the relatively simple near-Earth asteroid thermal model (NEATM, Harris 1998) to fit our 5 to $13 \mu \mathrm{m}$ spectrum. The NEATM is a refinement of the standard thermal model (STM, Lebofsky et al. 1986; Lebofsky \& Spencer 1989), which was developed and calibrated for main-belt asteroids. Unlike the STM, the NEATM requires observations at multiple wavelengths and uses this information to force the model 

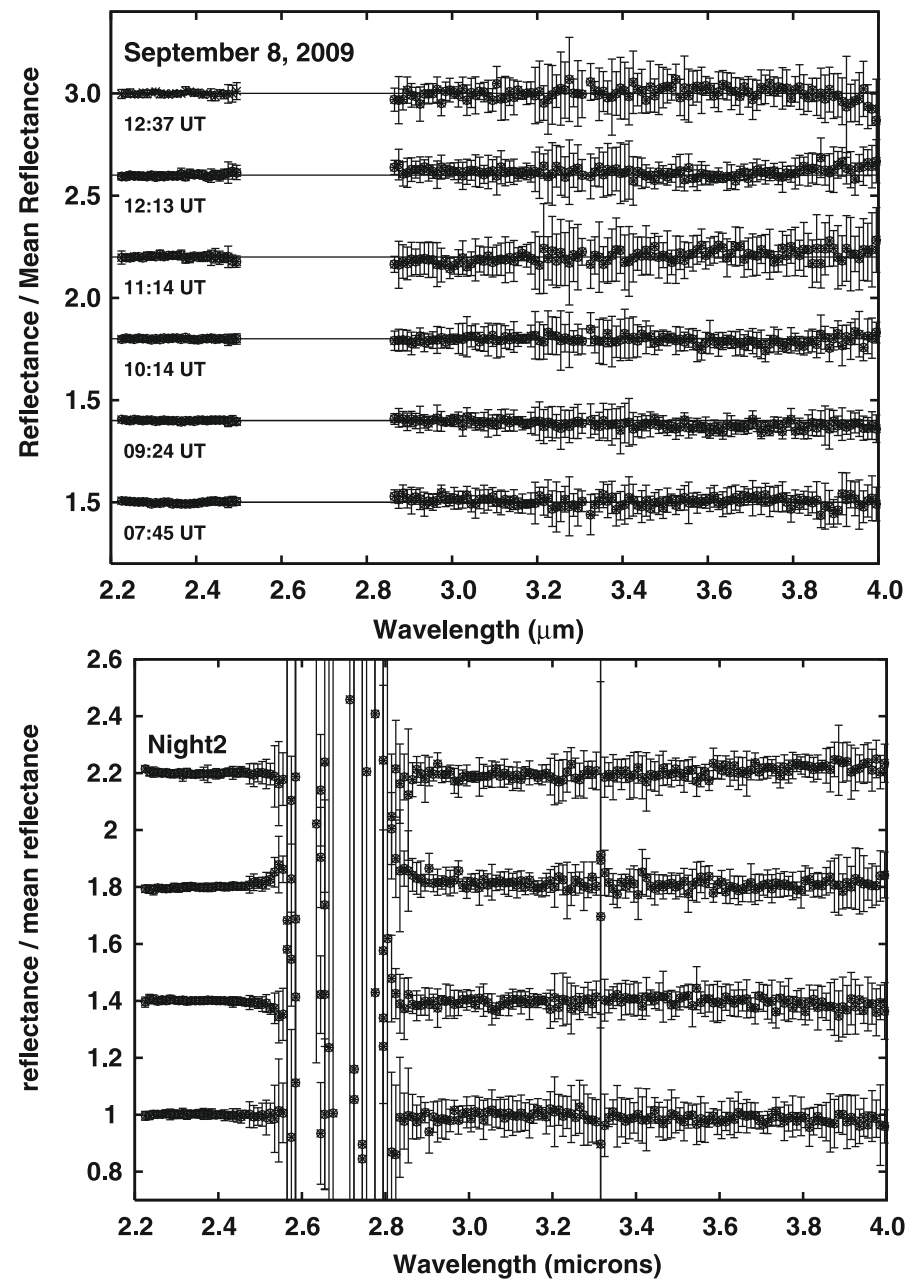

Fig. 3. IRTF spectra of Cybele divided by the mean spectrum of each night, shifted in reflectance for clarity. Notice that no significant differences are shown within the $S / N$.

temperature distribution to be consistent with the apparent color temperature of the asteroid. The NEATM solves simultaneously for the beaming parameter $(\eta)$ and the diameter $(D)$. The beaming parameter was originally introduced in the STM to allow the model temperature distribution to fit the observed enhancement of thermal emission at small solar phase angles due to surface roughness. In practice, $\eta$ can be thought of as a modeling parameter that allows a first-order correction for any effect that influences the observed surface temperature distribution (such as beaming, thermal inertia, and rotation).

We did not make any observations of this asteroid's reflected continuum below $2 \mu \mathrm{m}$, so to estimate the visible geometric albedo $\left(p_{V}\right)$, we adopted the values retrived from the JPL Small-Body Database Browser (http://ssd.jpl.nasa. gov /) of $H_{V}=6.62 \pm 0.12$ and $G=0.01 \pm 0.009$, where $H_{V}$ is the visible absolute magnitude and $G$ is the slope parameter. Using the NEATM (with bolometric emissivity 0.9 ) we derived the following: $D=290 \pm 5 \mathrm{~km}, \eta=0.967 \pm 0.014$, and $p_{V}=0.05 \pm 0.01$. The best-fit NEATM is shown in Fig. 1.

Thermal observations of Cybele have been published in several papers (Tedesco \& Veeder 1992; Tedesco et al. 2002; Morrison \& Chapman 1976; Morrison 1977; Bowell et al. 1979; Green et al. 1985; Müller \& Blommaert 2004). Müller \& Blommaert (2004) analyzed all of them and using thermophysical models, derived for Cybele a size of $302 \times 290 \times 232 \mathrm{~km}$

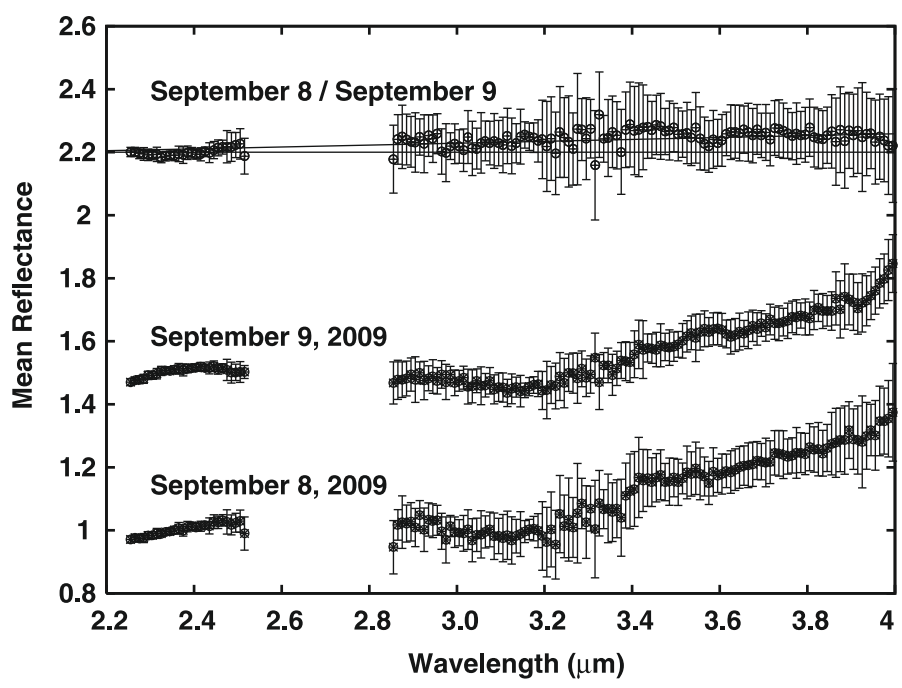

Fig. 4. Mean spectrum of Cybele obtained on September 8 and 9, shifted in reflectance for clarity, and the division of both (also shifted). Notice that the differences are only significant in the slope, the September 9 spectrum being slightly redder by about $0.5 \% / 0.1 \mu \mathrm{m}$.

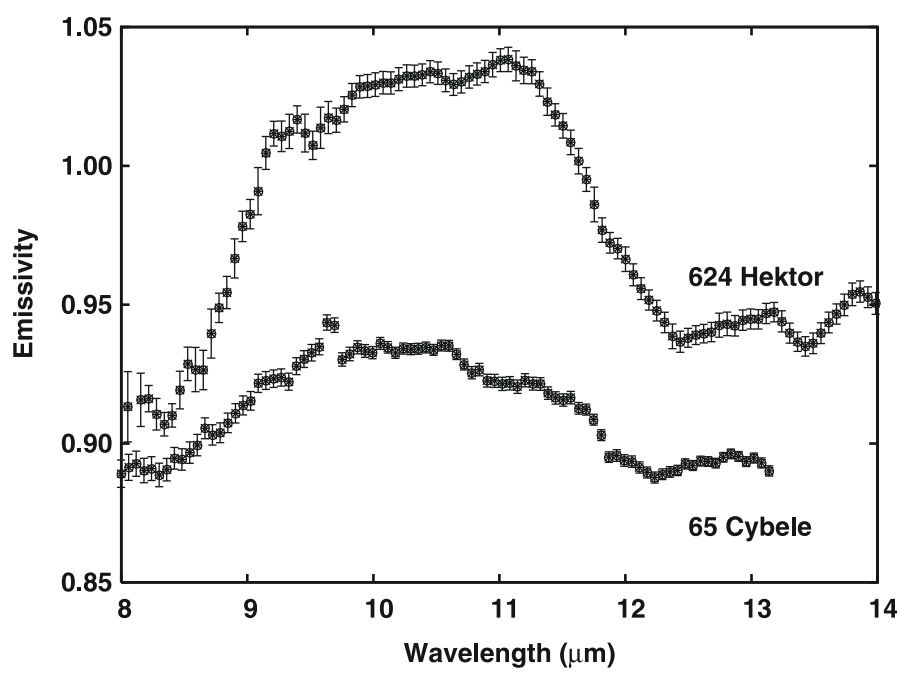

Fig. 5. Emissivity spectrum of Cybele compared to that of Trojan asteroid (624) Hektor (shifted in the emissivity axis for clarity). Notice the emission plateau due to fine silicate grains in the spectrum of both asteroids. Notice also that the contrast is smaller $(\sim 5 \%)$ in the spectrum of Cybele than in that of Hektor $(\sim 15 \%)$.

$( \pm 4 \%)$, an effective diameter $D=273 \pm 11.9 \mathrm{~km}$, and a geometric visible albedo of $0.050 \pm 0.005$. The data presented in this paper has higher $S / N$ and spectral resolution in the $5-14 \mu \mathrm{m}$ spectral range, and the results are consistent with those of Müller \& Blommaert (2004).

\section{The emissivity spectrum}

The emissivity spectrum of Cybele was obtained by dividing the flux density spectrum by the obtained NEATM model and it is shown in Fig. 5. The spectrum clearly exhibits an emission plateau at about 9 to $12 \mu \mathrm{m}$ with a spectral contrast of $\sim 5 \%$. This emission resembles that of silicates (the $\mathrm{Si}-\mathrm{O}$ stretch fundamental).

The spectra of the three Trojan asteroids in Emery et al. (2006) also show a similar emission plateau with a highest contrast of 10 to $15 \%$. To illustrate that, the emissivity spectrum of 
Cybele is plotted in Fig. 5 together with that of Trojan asteroid (624) Hektor (from Emery et al. 2006).

Emery et al. (2006) present an extensive comparison of the Trojan's spectra with that of different kind of silicates and meteorite samples and with cometary dust comae, and concluded that fine-grained silicates, and no other mineral group reproduce the major features of the spectra. They argue that the emissivity spectra more closely resemble the emission spectra from cometary comae than those from solid surfaces and those measured in the laboratory for powdered meteorites and regolith analogs. They finally hypothesize that the coma-like emission from the solid surfaces of Trojans may be due to small silicate grains being imbedded in a relatively transparent matrix, or to a very under-dense ("fairy-castle") surface structure. Another possibility for the fine-grained silicate emission features is the electrostatic levitation of small particles above the surface (Lee 1996), thus producing a dispersed medium. The similarities between Cybele and Trojan asteroids spectra suggest that the proposed emission mechanism for the Trojans are also valid for this asteroid. The lower amplitude of the silicate emission in Cybele spectrum respect to that of Hektor can be attributed to larger dust particles and/or a slightly denser structure.

\section{The near-infrared spectrum in the $3 \mu \mathrm{m}$ spectral region}

Longward of about $3.3 \mu \mathrm{m}$ the LXD spectrum contains a thermally emitted component. This thermal component was modeled using the thermal model derived from the Spitzer spectrum (see Sect. 3) and subtracted from the mean IRTF spectrum to properly define the shape of the reflected continuum beyond $3.3 \mu \mathrm{m}$. The final reflectance spectrum was transformed to albedo units (Fig. 6) by scaling the reflectance at $2.4 \mu \mathrm{m}$ to an albedo of 0.06 which results from the fact that Cybele has a linear spectrum in the $0.5-2.4 \mu \mathrm{m}$ range with an spectral slope $S_{V}^{\prime}=0.95 \% / 1000 \AA$ (Licandro et al. 2008).

Cybele's spectrum presents a deep absorption band centered at $\sim 3.1 \mu \mathrm{m}$. Notice that the thermal contribution shortward of $3.3 \mu \mathrm{m}$ is negligible (see Fig. 6); hence, the position and shape of the $3.1 \mu \mathrm{m}$ band are unaffected by the subtraction of the thermal continuum.

The spectrum of Cybele, and in particular the band center and shape of the $3.1 \mu \mathrm{m}$ absorption band, strongly resembles that of two other primitive asteroids in the outer edge of the main belt: Themis (Campins et al. 2010; Rivkin \& Emery 2010) and (375) Ursula (hereafter Ursula, see Fig. 10 of Rivkin et al. 2003). Cybele (with a semi-major axis $a=3.433 \mathrm{AU}$ ) is just outside the 2:1 resonance while Themis and Ursula (semi-major axis $a=$ 3.129 and 3.125 AU respectively) are just inside it.

Based on the spectra of Themis obtained with the IRTF, Campins et al. (2010) and Rivkin \& Emery (2010) report the first detection of water ice and complex organics on the surface of an asteroid, and they also show that the ice and the organics are widely distributed across the surface. In this paper, we use the shorthand term organics to mean the relatively refractory solid material consisting of complex macromolecular carbonaceous material that is similar to the insoluble organic matter found in most carbonaceous meteorites. This material contains both aromatic and aliphatic hydrocarbons, amorphous carbon, and other materials of undetermined structure. Tholins are synthetic complex organics and carbon nitrides that are often used to model surfaces of Solar System bodies, in part because their complex refractive indices have been measured, and because their use in

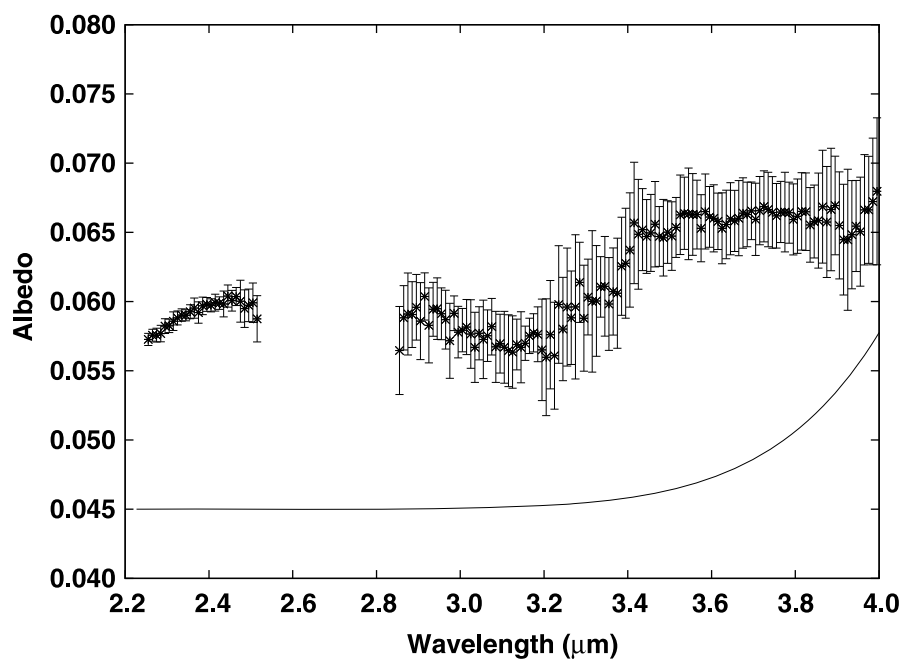

Fig. 6. Mean spectrum of Cybele in the $2-4 \mu \mathrm{m}$ range. The thermal contribution was subtracted and the reflectance transformed to albedo units (see text). The thermal contribution is shown as a solid line (scaled to albedo units and shifted by 0.045 to fit in the figure). Notice that the thermal contribution in the $3.1 \mu \mathrm{m}$ band is negligible.

scattering models often reproduces the reflectance spectra satisfactorily.

Analyzing the $\sim 3.1 \mu \mathrm{m}$ band Rivkin \& Emery (2010) first conclude that its shape is unlike those seen in hydrated minerals, carbonaceous chondrites or typical C-class asteroids. They attribute the band to fine-grained water ice as a frost deposited on regolith grains and model the ice feature using an scattering model that include a thin coating of $\mathrm{H}_{2} \mathrm{O}$ ice on surface grains (see Rivkin \& Emery 2010, for details). In Fig. 7 we compare Themis and Cybele spectra. We also overplot on the spectrum of Themis the spectral model included in Fig. 1 of Rivkin \& Emery (2010) and a model with similar characteristics and composition is overplotted on Cybele's spectrum. The model for Cybele consist of an intimate (salt-and-pepper) mixture of $22 \%$ amorphous pyroxene with $0.04 \mu \mathrm{m}$ thick rims of $\mathrm{H}_{2} \mathrm{O}$ and $78 \%$ amorphous carbon. Both materials have grain sizes of $30 \mu \mathrm{m}$ and the surface porosity is 0.31 . The differences from the parameters used for the model of Themis's spectrum (see Rivkin \& Emery 2010, for details) are not significant. In both cases the models fit very well at short wavelengthes while at longer wavelengths, beyond $3.2 \mu \mathrm{m}$ they do not.

The residuals of dividing Themis spectrum against the model show evidence of additional spectral structure (see Fig. 2 of Rivkin \& Emery 2010) that can be explained by various organic material. Campins et al. (2010, supplementary information) fit the spectrum of Themis in the 3.0 to $3.6 \mu \mathrm{m}$ spectral range with a model containing a small amount of very fine grains of water ice and ice tholins, obtaining a good fit of the absorption bands due to organics in the 3.2 to $3.5 \mu \mathrm{m}$ spectral range. The residuals of dividing Cybele's spectrum against the model are shown in Fig. 8 together with the spectra of organics with a different compositions. The three organic compounds plotted in Fig. 8 are: 1) a sample of Insoluble Organic Material (Orgueil IOM); 2) an average of six polycyclic aromatic hydrocarbons; 3 ) ice tholin.

The first case, the spectrum of the sample of Insoluble Organic Material (Orthous-Dauna et al. 2010) shows the contribution of the aliphatic structure. The 3.279-3.636 microns range is dominated by $\mathrm{C}-\mathrm{H}$ stretching modes (symmetric and asymmetric). The aliphatic structure is given by the relative contribution of methyl $\left(\mathrm{CH}_{3}\right)$, methylene $\left(\mathrm{CH}_{2}\right)$ bands and tertiary 


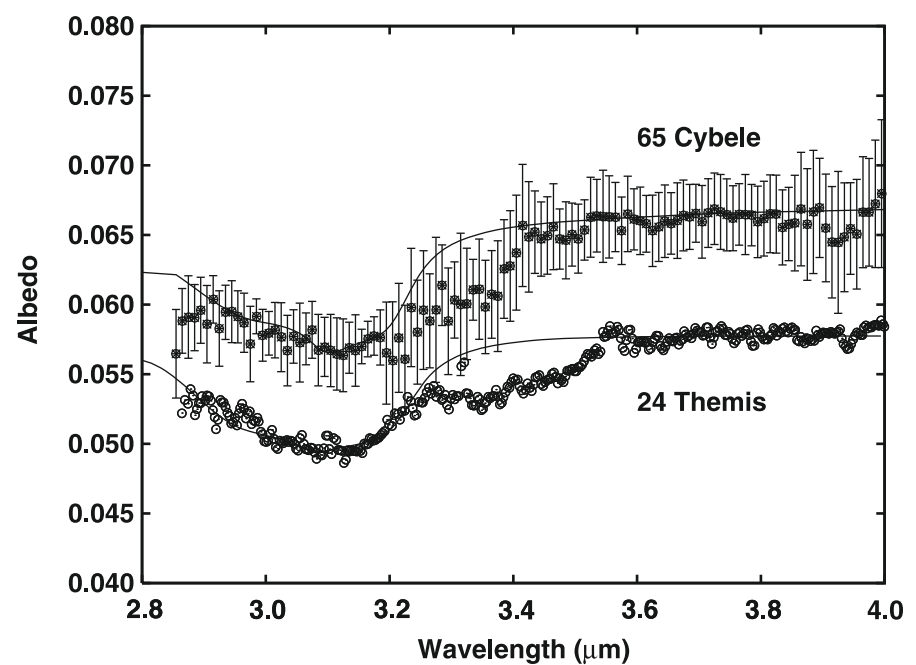

Fig. 7. Reflectance spectrum of Cybele and Themis in albedo units. Within the noise, both spectra looks very similar. Overplotted on the spectrum of Themis is the spectral model included in Fig. 1 of Rivkin \& Emery (2010). A similar model is overplotted on Cybele's spectrum. These models include a thin coating of $\mathrm{H}_{2} \mathrm{O}$ ice on surface grains. Notice that models fit very well the left part of the band while at longer wavelengths there are additional absorptions.

hydrogen $(\mathrm{CH})$. The second case, the average spectrum of six polycyclic aromatic hydrocarbons taken from Colangeli et al. (1992) shows absorption features in organic materials near $3.3 \mu \mathrm{m}$ that corresponds to the aromatic stretching bands. The third case, the ice tholin is the residual of an irradiated mixture of water ice and ethane. The spectrum was obtained using the Skhuratov scattering theory (Shkuratov et al. 1999) from optical constants taken from Khare et al. (1993). The ice tholin combines the contributions of both, aliphatic and polycyclic aromatic hydrocarbons.

Notice that the shape of the residuals in Cybele's spectrum is different from that of the residuals in Themis spectrum, but they can both be explained by a combination of organic material like those shown in Fig. 8. The observed difference between the spectrum of Themis and Cybele is indicative of a difference in the composition of the organics in both asteroids, which is also compatible with the difference in the observed color of the visible and near-infrared spectra (Cybele's spectrum is redder than that of Themis spectrum). A detailed study of these bands could be used to obtain information on the organics that are present on their surfaces, but, at least in the case of Cybele, the $S / N$ is not sufficient to do that.

So, from the $3 \mu \mathrm{m}$ spectra of Cybele presented in this paper, we conclude that its surface likely has fine-grained water ice as a frost deposited on regolith grains and complex organic solids, both over most of its surface. Notice also that no hydrated silicates are detected suggesting that most of the silicates in the surface of this asteroid are anhydrous.

Finally notice that Themis, Cybele, and Ursula, the only Cclass asteroid with semi-major axis $>3.1$ AU observed in spectroscopic mode in the $2-4 \mu \mathrm{m}$ spectral range with high enough $S / N$, do not present the typical absorption band due to hydrated sylicates. This is in agreement with Jones et al. (1990) that suggest that the C-type asteroids underwent a widespread, though mild, heating and aqueous alteration episode, and that the hydrated silicate abundance declines gradually among them from 2.5 to $3.5 \mathrm{AU}$.

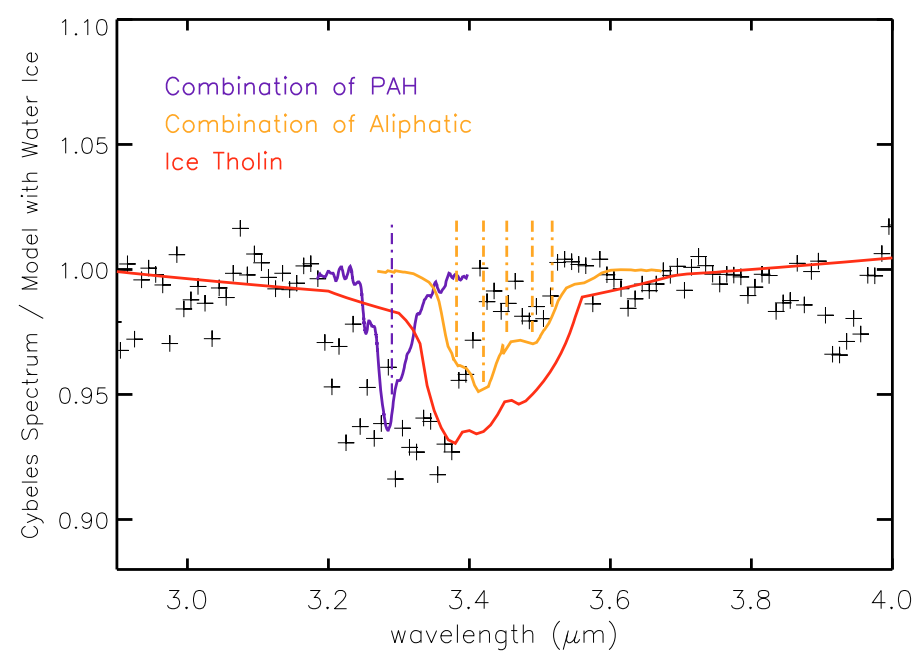

Fig. 8. The residuals of dividing Cybele's spectrum against the model are shown (as crosses) together with the spectra of organics with different composition. In yellow is the spectrum of a sample of Insoluble Organic Material from Orthous-Dauna et al. (2010) showing the contribution of the aliphatic structure. The purple line is the spectrum of an average of six polycyclic aromatic hydrocarbons from Colangeli et al. (1992). The red line is the spectrum of ice tholin from Khare et al. (1993).

\section{Discussion and conclusions}

In this paper we present near-infrared $2-4 \mu \mathrm{m}$ and mid-infrared 5-14 $\mu \mathrm{m}$ spectroscopy of Cybele obtained with the NASA IRTF telescope and the Spitzer space observatory, and yield the following results:

1. The 5-14 $\mu \mathrm{m}$ spectrum was analyzed using NEATM and we derived a diameter $D=290 \pm 5 \mathrm{~km}, \eta=0.967 \pm 0.014$, and $p_{V}=0.05 \pm 0.01$. The albedo of Cybele is low, typical of a C-type asteroids, and similar to the albedo of large Trojan asteroids (Fernández et al. 2003), comet nuclei (e.g. Campins et al. 2006), and to the albedo of Themis and Themis family members (Hsieh et al. 2009).

2. The emisivity spectrum in the 5-14 $\mu \mathrm{m}$ spectral range exhibits an emission plateau at about 9 to $12 \mu \mathrm{m}$ with a spectral contrast of $\sim 5 \%$. The observed spectral emissivity is similar to that observed in Trojan asteroids (Emery et al. 2006) which is attributed to small silicate grains being imbedded in a relatively transparent matrix, or to a very under-dense (fairy-castle) surface structure. Alternatively, electrostatic levitation of small particles above the surface can produce also a dispersed medium. The lower amplitude of the silicate emission in Cybele spectrum respect to that of Trojan asteroids can be attributed to larger dust particles and/or a slightly denser structure. Notice that a "dusty" surface like that of Trojans and Cybele is similar to how one would expect a cometary dust mantle to look like.

3. From the presented 2-4 $\mu \mathrm{m}$ spectra of Cybele, we report the detection of an absorption band centered at $3.1 \mu \mathrm{m}$, similar to that in the spectrum of Themis (Campins et al. 2010; Rivkin \& Emery 2010) that can be modeled using water ice. We conclude that its surface has fine-grained water ice as a frost deposited on regolith grains and complex organic solids that are spread over its whole surface.

4. Other absorption bands in the 3.2-3.6 $\mu \mathrm{m}$ region indicate the presence of complex organic solids on the surface, as in the case of Themis. The observed difference between the 
spectrum of Themis and Cybele is indicative of a difference in the composition of the organics, which is also compatible with the observed difference in the observed color of both asteroids in the visible and near-infrared where Cybele is redder than Themis spectrum.

5. No hydrated silicates are detected in the $2-4 \mu \mathrm{m}$ spectra of Cybele, suggesting that most of the silicates in the surface of this asteroid are anhydrous. This is also consistent with the structure observed in the $7-14 \mu \mathrm{m}$ spectrum. The similarity in the $2-4 \mu \mathrm{m}$ spectra of the outer belt asteroids Cybele, Themis and Ursula is in agreement with Jones et al. (1990) that suggest that the hydrated silicate abundance in C-class asteroids declines with distance to the Sun.

6. Cybele's surface is covered by a fine anhydrous silicate grains, with a small amount of water ice and complex organic solids. This is similar to comet surface were non-equilibrium phases coexist, e.g. water ice and anhydrous silicates, thus we conclude that this is a very primitive object. The presence of water-ice and anhydrous silicates is indicative that hydration has not occured or is incomplete, suggesting that the temperatures were always sufficiently slow.

7. The surface of Cybele has similarities to that of Themis and to the surface of Trojan asteroids. More studies are needed to determine any possible link between Themis family, Cybeles and Trojan asteroids. For example, higer $S / N$ spectra of Trojan asteroids in the $2-4 \mu \mathrm{m}$ region than those in Emery et al. (2006) are needed to detect possible water ice and organic absorptions like those reported in Themis and Cybele.

Acknowledgements. We thanks Dr. Antonella Barucci for her comments on the manuscript. J. L. gratefully acknowledges support from the spanish "Ministerio de Ciencia e Innovación" (MICINN) project AYA2008-06202-C03-02. H. C. gratefully acknowledges support from NASA Spitzer Science Center, Jet Propulsion Laboratory and Planetary Astronomy program. This research was partially funded by the MICINN through CSD2006-00070. N. P. acknowledges support from the NASA Postdoctoral Program at the Ames Research Center, administered by Oak Ridge Associated Universities through a contract with NASA.

\section{References}

Abell, P., Fernández, Y., Pravec, P., et al. 2005, Icarus, 179, 174

Barucci, M. A., Capria, M. T., Coradini, A., \& Fulchignoni, M. 1987, Icarus, 72, 304

Bowell, E., Gehrels, T., \& Zellner, B. 1979, in Asteroids, ed. T. Gehrels (University of Arizona Press), 1108

Bus, S. J. 1999, Ph.D. Thesis, Massachusetts Institute of Technology

Campins, H., Ziffer, J., Licandro, J., et al. 2006, AJ, 132, 1346

Campins, H., Ziffer, J., Licandro, J., et al. 2007, AJ, 132, 1346

Campins, H., Hargrove, K., Pinilla-Alonso, N., et al. 2010, Nature, 464, 1320
Cohen, M., Witteborn, F. C., Roush, T., Bregman, J., \& Wooden, D. 1998, AJ, 115,1671

Colangeli, L., Mennella, V., Baratt, G. A., Bussoletti, E., \& Strazzulla, G. 1992, ApJ, 396, 369

Cushing, M. C., Vacca, W. D., \& Rayner, J. T. 2004, PASP, 116, 362

DeMeo, F., Binzel, R., Silvan, S., \& Bus, S. 2009, Icarus, 202, 160

Emery, J. P., \& Brown, R. H. 2003, Icarus, 164, 104.

Emery, J. P., \& Brown, R. H. 2004, Icarus, 170, 131

Emery, J. P., Cruikshank, D. P., \& van Cleve, J. 2006, Icarus, 182, 496

Fernández, J. 1980, MNRAS, 192, 481

Fernández, Y., Sheppard, S., \& Jewitt, D. 2003, AJ, 126, 1563

Gil-Hutton, R. 1990, MP Bulletin, 17, 34

Green, S. F., Eaton, N., Aitken, D. K., et al. 1985, Icarus, 62, 282

Harris. A. W. 1998, Icarus, 131, 291

Hsieh, H., \& Jewitt, D., 2006, Science, 312, 561

Hsieh, H., Jewitt, D., \& Fernández, Y. 2009, ApJ, 694, 111

Jones, T., Lebofsky, L., Lewis, J., \& Marley, M. 1990, Icarus, 88, 172

Khare, B. N., Thompson, W. R., Cheng, L., et al. 1993, Icarus, 103, 290

Lamy, P., Toth, I., Fernández, Y., \& Weaver, H. 2004, in Comets II, ed. M. C.

Festou, H. U. Keller, \& H. A. Weaver (Tucson: University of Arizona Press), 223

Landolt, A. 1992, AJ, 104, 340

Lazzaro, D., Angeli, C., Carvano, J., et al. 2004, Icarus, 172, 179

Lee, P. 1996, Icarus, 124, 124

Lebofsky, L. 1980, AJ, 85, 873

Lebofsky, L. A., \& Spencer, J.R., 1989, in Asteroids II, ed. R. P. Binzel, T. Gehrels, \& M. S. Matthews (Tucson: Univ. of Arizona Press), 128

Lebofsky, L. A., Sykes, M. V., Tedesco, E. F., et al. 1986, Icarus, 68, 239

Levison, H., Bottke, W., Gounelle, M., et al. 2009, Nature, 460, 364

Licandro, J., Campins, H., Hergenrother, C., \& Lara, L. M. 2002, A\&A, 398, L45

Licandro, J., Alvarez-Candal, A., de Len, J., et al. 2008, A\&A, 481, 861

Lim, L., McConnochie, T., Bell, J.n \& Hayward, T. 2005, Icarus, 173, 385

Lord, S. 1992, A new software tool for computing Earths atmospheric transmission of near and far-infrared radiation, NASA Technical Memo. 103957

Morbidelli, A., Levison, H. F., Tsiganis, K., \& Gomes, R. 2005, Nature, 435, 462

Morrison, D. 1977, ApJ, 214, 667

Morrison, D., \& Chapman, C. R. 1976, ApJ, 204, 934

Müller, T., \& Blommaert, J. 2004, A\&A, 418, 347

Orthous-Daunay, F., Quirico, E., Beck, P., Brissaud, O., \& Schmitt, B. 2010, in 41st Lunar and Planetary Science Conference LPI Contribution No. 1533, 1803

Rayner, J. T., Toomey, D. W., Onaka, P. M., et al., 1998, SPIE, 3354, 468

Rivkin, A. S., \& Emery, J. 2010, Nature, 464, 1322

Rivkin, A. S., Howell, E. S., Vilas, F., \& Lebofsky, L. A. 2002, in Asteroids III, ed. W. F. Bottke, A. Cellino, P. Paolicchi, R. P., \& Binzel (Univ. Arizona Press), 235

Rivkin, A. S., Davies, J., Johnson, J, et al. 2003, Meteoritic \& Planetary Science 38,1383

Shkuratov, Y., Starukhina, L., Hoffmann, H., \& Arnold, G. A. 1999, Icarus, 137, 235

Tedesco, E. F., Noah, P. V., Noah, M., \& Price, S. D. 2002, AJ, 123, 1056

Tedesco, E. F., \& Veeder, G. J. 1992, The IRAS Minor Planet Survey, Phillips Laboratory, 243

Tholen, D. J. 1989, in Asteroids II, ed. R. P. Binzel, T. Gehrels, M. S. Matthews (Tucson: Univ. of Arizona Press), 298

Volquardsen, E. L., Rivkin, A. S., \& Bus, S. J. 2007, Icarus, 187, 464 